\title{
Cloning and Expression of Bovine Sodium/Glucose Cotransporters*
}

\author{
F.-Q. Zhao, Y.-C. Zheng, E. H. Wall, and T. B. McFadden \\ Lactation and Mammary Gland Biology Group, \\ Department of Animal Science, University of Vermont, Burlington 05405
}

\section{ABSTRACT}

The $\mathrm{Na}^{+}$-dependent glucose cotransporters (SGLT) are a family of glucose transporters that mediate an active, sodium-linked transport process against an electrochemical gradient. The SGLT are known to play important roles in absorption of dietary D-glucose and D-galactose from the intestinal lumen and in the reabsorption of $\mathrm{D}$ glucose from the glomerular filtrate in kidney. To study the role and regulation of SGLT in tissues of lactating cows, we cloned and sequenced the full-length cDNA of bovine SGLT1 and SGLT5. Open reading frame analysis predicted that bovine SGLT1 is composed of 664 amino acids with a molecular weight of approximately $73 \mathrm{kDa}$, and SGLT5 is composed of 597 amino acids with a molecular weight of approximately $65 \mathrm{kDa}$. The deduced amino acid sequence of bovine SGLT1 is $48 \%$ identical and $66 \%$ conserved relative to that of bSGLT5. The amino acid sequence of bovine SGLT1 is $97,88,87,86,85$, and $84 \%$ identical to sheep, mouse, rat, horse, human, and rabbit SGLT1, respectively. In contrast, the amino acid sequence of bSGLT5 is relatively divergent among species, being 85,64 , and $48 \%$ identical to rabbit, human, and rat SGLT5, respectively. Bovine SGLT retain the characteristic structural features of SGLT1 proteins described in other species, including membrane-spanning helices and glucose transporter motifs. The major in vitro transcription and translation product of bovine SGLT1 cDNA migrated at an apparent molecular weight of $52 \mathrm{kDa}$. In the presence of canine microsomal membranes, the translation product increased to $53 \mathrm{kDa}$, suggesting glycosylation. The SGLT1 mRNA was most abundant in bovine intestine, at intermediate levels in bovine kidney, and at lower levels in bovine mammary gland, liver, and lung. No SGLT1 mRNA was detected in bovine spleen, skeletal muscle, or testes. Expression of SGLT5 mRNA was found predominantly in bovine kidney, only at very low levels in bovine testes, skeletal muscle, and spleen,

\footnotetext{
Received June 25, 2004.

Accepted October 6, 2004.

Corresponding author: Feng-Qi Zhao; e-mail: fzhao@uvm.edu.

*The nucleotide sequence data reported in this paper have been submitted to GenBank with assigned accession numbers AF508807 (bSGLT1) and AY514442 (bSGLT5).
}

and was essentially undetectable in bovine mammary gland, liver, lung, and small intestine. Abundance of SGLT1 mRNA in bovine mammary gland increased more than 4-fold during late pregnancy and early lactation. The sequence and expression data reported in this paper lay the groundwork for future studies aimed at unraveling the functional roles of SGLT in supporting milk production and maintaining glucose homeostasis during lactation.

(Key words: gene expression, glucose transport, glucose uptake, mammary gland)

Abbreviation key: bSGLT1 and bSGLT5 = bovine solute carrier family 5 (sodium/glucose cotransporter) member 1, and 5 (gene symbols SLC5A1 and SLC5A5), $\operatorname{poly}(\mathbf{A})^{+}=$polyadenylated $\mathrm{RNA}, \mathbf{R A C E}=$ rapid amplification of cDNA ends, SGLT = sodium/glucose cotransporter family, $\mathbf{T M}=$ transmembrane domain, $\mathbf{U T R}=$ untranslated region.

\section{INTRODUCTION}

Glucose transport across plasma membranes is mediated by 2 distinct families of glucose transporters (Wood and Trayhurn, 2003). In most tissues and cells, glucose is transported by a bidirectional and energy-independent process mediated by members of the facilitative glucose transporter family (solute carriers SLC2A, protein symbol GLUT). However, in a few cell types, glucose is transported by an active, sodium-linked transport process against an electrochemical gradient. This process is mediated by the sodium-dependent glucose transporters or $\mathrm{Na}^{+} /$glucose cotransporters (solute carriers SLC5A, protein symbol SGLT), and is believed to be restricted to the brush-border membranes of small intestine and proximal convoluted tubule of the kidney.

Thus far, 6 SGLT isoforms (SGLT1 to 6) have been cloned from various species (Wright and Turk, 2004). These transporters exhibit different substrate specificities, kinetic properties, and tissue expression profiles, indicating that each transporter plays a distinct role in maintaining body glucose homeostasis. In humans, SGLT1, a high affinity $\mathrm{Na}^{+} /$glucose cotransporter $\left(K_{m}=\right.$ $0.4 \mathrm{mM}$ ), is primarily located in the brush-border membrane of mature enterocytes of small intestine and is involved in absorption of dietary D-glucose and D-galac- 
tose from the intestinal lumen (Wright, 1993). SGLT2 is a low-affinity glucose transporter that is predominantly located on the apical membrane of renal proximal convoluted tubules, and is presumably responsible for the reabsorption of D-glucose from the glomerular filtrate (Wells et al., 1992; Kanai et al., 1994). SGLT3 was originally cloned as a renal amino acid cotransporter in swine (Kong et al., 1993) and was later reclassified as a member of the SGLT family (Mackenzie et al., 1994). However, its function may need to be reevaluated because human SGLT3 apparently does not transport glucose (Wright and Turk, 2004). Little is known about the isoforms SGLT4 to 6 , except that SGLT4 and 6 are expressed in a wide variety of tissues, whereas expression of SGLT5 is restricted to kidney (Wright and Turk, 2004).

In our previous studies, we reported that SGLT1 is expressed in the mammary gland and in ruminal and omasal mucosa of lactating cows (Zhao et al., 1998, 1999). The expression of SGLT1 in the mammary gland suggests that an active glucose-transport system may be involved in transport and metabolism of glucose in this tissue. This contradicts the prevailing opinion that glucose is transported into mammary cells solely through a passive process of facilitated diffusion (Xiao and Cant, 2003). The relatively high expression of SGLT1 in ruminal and omasal mucosa implies new functional roles for these tissues in glucose absorption and nutrition. To further study the physiological roles of SGLT1 in the mammary gland and forestomach of lactating cows, we cloned the full-length cDNA of bovine SGLT1 and studied the tissue distribution of its mRNA expression. We also cloned the full-length cDNA of bovine SGLT5 because these 2 transporters share high sequence homology. We report here that the expression of SGLT1 is developmentally regulated in bovine mammary gland in a manner consistent with a role in milk synthesis.

\section{MATERIALS AND METHODS}

\section{Animals, Tissues, and RNA Isolation}

Unless otherwise specified below, the mammary gland, liver, kidney, lung, spleen, jejunal epithelia, and skeletal muscle tissues were obtained from 2 lactating (185 and $381 \mathrm{~d}$ in lactation, respectively) Holstein cows (Bos taurus), and the testicular tissue from 1 Holstein bull, immediately after slaughter in a local abattoir. Collection of tissues was approved by the University of Vermont Institutional Animal Care and Use Committee. The tissue samples were quickly frozen in liquid $\mathrm{N}_{2}$, transferred to the laboratory, and stored at $-80^{\circ} \mathrm{C}$ until used for preparation of RNA. Total RNA was isolated from each tissue using TRIzol reagent (Invitrogen, Carlsbad, CA). Polyadenylated [poly(A) $\left.{ }^{+}\right]$RNA was isolated from total RNA pooled from the 2 lactating cows using oligodeoxythymidylate cellulose chromatography (Jacobson, 1987).

\section{Rapid Amplification of cDNA Ends and Cloning of bSGLT1 and bSGLT5}

The sequences of all primer oligonucleotides used in this study are listed in Table 1.

The $3^{\prime}$ and $5^{\prime}$ sequences of bovine solute carrier family 5 (sodium/glucose cotransporter) member 1 and 5 (bSGLT1 and bSGLT5) were obtained by rapid amplification of cDNA ends (RACE) using the SMART RACE cDNA amplification kit (Clontech, Palo Alto, CA). The 3' and 5' RACE-ready first-strand cDNA were synthesized using $1 \mu \mathrm{g}$ of poly(A) ${ }^{+}$RNA from bovine kidney. For $5^{\prime}$ RACE of bSGLT1, 2 primers, SGLT-R1 and SGLT-R2, were originally designed at highly conserved regions of SGLT1 based on the sequence alignment of human, mouse, rat, horse, and sheep SGLT1 cDNA. Our 5' RACE experiment using the provided universal primer (UPM, Clontech) and SGLT-R2 resulted in $5^{\prime}$ sequence of bSGLT1. However, the RACE using UPM and SGLT$\mathrm{R} 1$ resulted in $5^{\prime}$ sequence of bSGLT5, presumably due to high sequence homology between the 2 transporters. The 3 ' sequences of bSGLT1 and bSGLT5 were amplified using the primers UPM and SGLT-F2 designed from the highly conserved region of SGLT1 cDNA among species or bSGLT5-880F designed based on our 5' RACE data, respectively. The resulting PCR products were gel-purified, cloned into pCR 2.1 vector (Invitrogen) and sequenced using an ABI 377 automated sequencer (Applied Biosystems, Foster City, CA). The sequences of $3^{\prime}$ and $5^{\prime}$ RACE products were verified in at least 3 independent clones.

Bovine full-length cDNA of SGLT1 was amplified from the bovine kidney $5^{\prime}$ RACE-ready first-strand cDNA by PCR using $p f u$ polymerase (Stratagene, La Jolla, CA) with primers of bSGLT1-10F and bSGLT1-R3 designed from the $5^{\prime}$ - and $3^{\prime}$-untranslated regions (UTR) of bSGLT1 based on our RACE results. The PCR products were gel-purified and cloned into pCR-Blunt II-TOPO vector using Zero Blunt TOPO PCR Cloning Kit (Invitrogen) to form bSGLT1/pCR-Blunt II-TOPO. The fulllength bSGLT1 cDNA was sequenced, verified in at least 3 independent clones, and submitted to GenBank with accession number AF508807.

The full-length cDNA encoding bovine SGLT5 was first amplified from the bovine kidney $3^{\prime}$ RACE-ready first-strand cDNA above by PCR using Herculase-enhanced DNA polymerase (Stratagene) with primers UPM and bSGLT5-32F designed from the 5'-UTR of bSGLT5 based on our 5'-RACE results. The reaction was reamplified using bSGLT5-32F and bSGLT5-R3 designed from the 3'-UTR of bSGLT5 based on our 3' RACE 
Table 1. Sequences of oligonucleotide primers used for PCR and rapid amplification of cDNA ends (RACE).

\begin{tabular}{|c|c|c|}
\hline Primer name & Type & Sequence \\
\hline SGLT-R1 & Reverse & 5'-GTAGGCGATGTTGGTACAGC-3' \\
\hline SGLT-R2 & Reverse & 5'-CGGAAGATGTGGAAGGAGTC-3' \\
\hline SGLT-F2 & Forward & 5'-GCCATGAAGCTGAAGATGAC-3' \\
\hline bSGLT5-880F & Forward & 5'-GGTGCACTGACCAGGTCATC-3' \\
\hline bSGLT1-10F & Forward & 5'-AGCTGCCACTATGGACAGTA-3' \\
\hline bSGLT1-R3 & Reverse & 5'-CCTTCTGCAGCTCAGATATG-3' \\
\hline bSGLT5-32F & Forward & 5'-TCTGCTGCAGGATGGCTGTG-3' \\
\hline bSGLT5-R3 & Reverse & 5'-CTCCTACTTGGCTGGCTTGG-3' \\
\hline bSGLT5-1506F & Forward & 5'-GGCTGGTGCTGGAGTTCTTG-3' \\
\hline bSGLT5-R2 & Reverse & 5'-CAGCGTCAGGCGAAGTAGGT-3' \\
\hline$\beta$ Actin-F & Forward & 5'-TAGACTTCGAGCAGGAGATG-3' \\
\hline$\beta$ Actin-R & Reverse & 5'-CCACCAGACAGCACTGTGTT-3' \\
\hline
\end{tabular}

data. The PCR products were then gel-purified, polished by Taq polymerase, and cloned into pCR 4-TOPO vector (Invitrogen). The bSGLT5 sequence of the resulting plasmid bSGLT5/pCR4-TOPO was sequenced, verified in at least 3 independent clones, and submitted to GenBank with accession number AY514442.

\section{DNA Sequence Analysis}

The analysis of cDNA sequences was conducted using the computer programs of DNAstar (DNASTAR, Madison, WI), the National Center for Biotechnology Information (NCBI) BLAST site (http://www.ncbi.nlm.nih.gov/ BLAST/), and the SWISS-PROT Scan-Prosite program (http://us.expasy.org/tools/scanprosite/). The multiple sequence alignment was performed with CLUSTAL W (open gap cost 10) (Thompson et al., 1994). The hydropathy plots of bSGLT1 and bSGLT5 were analyzed according to the algorithm of Kyte and Doolittle (1982), using the ProtScale program on the ExPASy proteomics server (http://us.expasy.org/cgi-bin/protscale.pl), using a 19-amino acid window.

\section{In Vitro Transcription/Translation}

The cDNA of bSGLT1 was excised from the plasmids bSGLT1/pCR-Blunt II-TOPO and subcloned into the vector plasmid pcDNA3.1(-) (Invitrogen) to form plasmid bSGLT1/pcDNA3.1. This plasmid was transcribed by T7 polymerase and translated in the presence of $\mathrm{L}$ $\left[{ }^{35}\right.$ S]methionine (Amersham, Piscataway, NJ) using the TNT coupled reticulocyte lysate system (Promega, Madison, WI). Reactions were performed in the presence or absence of canine microsomal membranes (Promega). The translation products were resolved by $12 \%$ (wt/vol) SDS-PAGE with the addition of $3 M$ urea to the gel and loading buffer to reduce membrane protein aggregation, then imaged using a phosphor-capture screen and ImageQuant software on a Storm PhosphorImager (Molecular Dynamics, Sunnyvale, CA).

\section{Reverse Transcription-PCR}

The total RNA from each tissue above was treated with RNase-free DNase I (Invitrogen), and $5 \mu \mathrm{g}$ of each resulting RNA sample was used to synthesize the firststrand cDNA using M-MLV reverse transcriptase (200 $\mathrm{U}$; Invitrogen) and oligo (dT) $12-18$ primer (Invitrogen) in a $40-\mu \mathrm{L}$ reaction. The first-strand $\mathrm{cDNA}(1 \mu \mathrm{L})$, along with $\mathrm{ddH}_{2} \mathrm{O}$ and DNase I-digested RNA from the mammary gland (negative controls) and $1 \mathrm{ng}$ of bSGLT1/pCRBlunt II-TOPO, bSGLT5/pCR4-TOPO plasmid DNA or pTRI- $\beta$-actin-mouse antisense control template (Ambion, Austin, TX) (positive controls), were then amplified by PCR in a final volume of $50 \mu \mathrm{L}$, containing $2.5 \mathrm{mM}$ $\mathrm{MgCl}_{2}, 0.2 \mathrm{mM}$ dNTP mixture, $5 \mu \mathrm{L}$ of $10 \times$ PCR buffer, 2.5 units of Taq DNA polymerase (Invitrogen), and 0.4 $\mu M$ of each primer. The primers used were SGLT-F2 and bSGLT1-R3 above for amplification of bSGLT1; bSGLT5$1506 \mathrm{~F}$ and bSGLT5-R2 for bSGLT5; $\beta$ Actin-F and $\beta$ Actin- $\mathrm{R}$ for $\beta$-actin (Table 1). An initial reaction of $5 \mathrm{~min}$ at $94^{\circ} \mathrm{C}$ was followed by 5 cycles of $30 \mathrm{~s}$ denaturation at $94^{\circ} \mathrm{C}, 30 \mathrm{~s}$ of annealing at $65^{\circ} \mathrm{C}$, and $1 \mathrm{~min}$ of extension at $72^{\circ} \mathrm{C} ; 25$ cycles of $30 \mathrm{~s}$ denaturation at $94^{\circ} \mathrm{C}, 30 \mathrm{~s}$ of annealing at $60^{\circ} \mathrm{C}$, and $1 \mathrm{~min}$ of extension at $72^{\circ} \mathrm{C}$; and a final extension step of $7 \mathrm{~min}$ at $72^{\circ} \mathrm{C}$. For each PCR reaction, $8 \mu \mathrm{L}$ was resolved on $1 \%$ agarose gels via electrophoresis. Photographs of the gels (stained with ethidium bromide) are shown in an inverted black/white format.

\section{Quantitative Reverse Transcription-PCR}

Mammary biopsies were obtained from 12 Holstein cows on $\mathrm{d}-40,-20,-7$, and +7 relative to expected calving ( \pm 5 d) using the protocol of Farr et al. (1996). On each respective day, biopsies were obtained from the same quarter of all 12 cows. Biopsies were obtained from the rear quarters, alternating between left and right rear quarters on alternate sampling days Total RNA was isolated from each biopsy sample, treated with deoxyri- 
bonuclease (DNase, Ambion) to remove contaminating DNA, and with RNA Secure (Ambion) to inactivate ribonucleases. Quality of RNA was verified by inspection of the 18S and 28S rRNA bands after agarose gel electrophoresis. The Stratascript II reverse transcription kit (Invitrogen) was used with oligo dT primers to reversetranscribe $5 \mu \mathrm{g}$ of total RNA into cDNA. A pool of undiluted cDNA of all samples was created and used to construct a standard curve including serial dilutions of stock, 1:5, 1:10, 1:100, and 1:1000, for determination of relative gene expression. To determine gene expression profiles, individual samples were diluted $1: 4$ and $1 \mu \mathrm{L}$ was amplified in a $25-\mu \mathrm{L}$ reaction mix containing 12.5 $\mu \mathrm{L}$ of SYBR Green Jumpstart Taq Ready Mix (Sigma, St. Louis, MO), along with $0.25 \mu \mathrm{L}$ of internal reference dye, and $0.1 \mu M$ each of forward and reverse gene-specific primer using an ABI 7700 (Taqman) instrument. The primers for amplifying bSGLT1 were SGLT-F2 and bSGLT1-R3. Optical data were collected at the end of each extension step, and mean expression relative to the common pool was determined. Data are reported as values normalized to the housekeeping gene, $\beta$-actin. Statistical significance of relative differences in mRNA expression was tested using the Mixed Procedure of SAS (version 8.2; SAS Institute, Inc., Cary, NC) to account for repeated measures. The model included biopsy day as a fixed effect and cow as a random variable. Differences between means at each stage of development were determined by Fisher's protected LSD test.

\section{RESULTS AND DISCUSSION}

\section{Isolation of the bSGLT1 and bSGLT5 cDNA by RACE}

Two primers (SGLT-R1 and SGLT-R2 as described under "Materials and Methods") were originally designed from highly conserved regions of SGLT1 based on the sequence alignment of human, mouse, rat, horse, and sheep SGLT1 cDNA to isolate the $5^{\prime}$ sequence of bovine SGLT1 cDNA by RACE. Our RACE experiments using SGLT-R2 resulted in the $5^{\prime}$ sequence of bSGLT1 cDNA, but those using SGLT-R1 resulted in a sequence highly homologous to the cDNA of a rabbit $\mathrm{Na}^{+}$/glucose cotransporter-related protein named RK-D (accession number U08813; Pajor, 1994), which was subsequently renamed SGLT5 (Wright and Turk, 2004). The 3' sequences of bSGLT1 and bSGLT5 were then obtained by RACE using different primers as described under "Materials and Methods". The 3' RACE of bSGLT1 revealed at least 3 transcripts with different lengths of the 3'-UTR (data not shown). Our previous study showed at least 4 different SGLT1 transcripts in bovine tissues (Zhao et al., 1999). The 3' RACE experiments in this study indicate that the different sizes of these transcripts are most likely due to different lengths of the $3^{\prime}$-UTR. The differences in patterns of SGLT1 transcripts between species and among different tissues in the same species (Zhao et al., 1999) imply that SGLT1 gene splicing or RNA editing mechanisms may differ across tissues and species.

The cDNA fragments comprising the full coding regions of bSGLT1 and bSGLT5 were isolated by RT-PCR with RNA from bovine kidney and primer oligonucleotides derived from the putative $5^{\prime}$ - and $3^{\prime}$-UTR of each gene (Figures 1 and 2). The sequence of each cDNA was verified in at least 3 independent clones and has been submitted to GenBank with accession numbers AF508807 (bSGLT1) and AY514442 (bSGLT5). Open reading frame analysis indicates that bovine SGLT1 is composed of 664 amino acids with a molecular weight of approximately $73 \mathrm{kDa}$, and SGLT5 is composed of 597 amino acids with a molecular weight of approximately $65 \mathrm{kDa}$.

Bovine SGLT1 and SGLT5 are highly related. The deduced amino acid sequence of bovine SGLT1 is $48 \%$ identical or $66 \%$ conservative to that of bSGLT5 (Figure 3). The major difference is that bSGLT1 has a much longer sequence in the region corresponding to the loop between the putative transmembrane domains 13 and 14 (see Figure 3). The amino-terminal (N-terminal) sequence also shares little homology.

The deduced amino acid sequence of bovine SGLT1 is $97,88,87,86,85$, and $84 \%$ identical or $99,93,93,92,94$, and $93 \%$ conservative to sheep (accession no. X82411), mouse (AF208031), rat (D16101), horse (AJ292081), human (M24847), and rabbit (X55355) SGLT1, respectively (Figure 4). The $\mathrm{N}$-terminus and the putative long loop 13 sequence are the most divergent regions across the species. The amino acid sequence of bSGLT5 is relatively divergent among species, with 85,64 , and $48 \%$ identity to or 91,70 , and $52 \%$ conservation with rabbit (U08813), human (AK057946), and rat (XM_220540) SGLT5, respectively (Figure 5). The striking differences among species are that rat SGLT5 has a 420 -amino acid sequence in the middle of the protein that is not present in other species, and human SGLT5 is about 100 amino acids shorter in the $\mathrm{N}$-terminus.

\section{Sequence Analysis of bSGLT1 and bSGLT5}

The hydropathy plot analysis of bovine SGLT1 and SGLT5 by ProtScale is consistent with the presumed tertiary structure of SGLT members (Turk and Wright, 1997; Wright and Turk, 2004), comprising 14 hydrophobic, and presumed membrane-spanning regions (TM) (Figure 6). The hydropathy plots of bSGLT1 and bSGLT5 are similar, having the same number and distribution of hydrophobic segments. However, there are striking 

ATGGACAGTAGCACCTGGAGCCCCCCGGCCACGGCCACCGCCGAGCCCCTCCCGCCCTACGAGCGCATCCGCAAT $\begin{array}{llllllllllllllllllllllllllll}M & D & S & S & T & W & S & P & P & A & T & A & T & A & E & P & L & P & P & Y & E & R & I & R & N\end{array}$ 76 GCAGCTGACATCTCTGTCATCGTCATCTACTTCGTGGTGGTGATGGCTGTCGGGCTGTGGGCCATGTTTTCCACT $\begin{array}{llllllllllllllllllllllllllll}A & A & D & I & S & V & I & V & I & Y & F & V & V & V & M & A & V & G & L & W & A & M & F & S & T\end{array}$

151 AACCGTGGGACCGTTGGAGGCTTCTTCCTGGCAGGTCGAAGTATGGTGTGGTGGCCGATTGGAGCCTCTCTGTTT $\begin{array}{llllllllllllllllllllllllllllll}N & R & G & T & V & G & G & F & F & L & A & G & R & S & M & V & W & W & P & I & G & A & S & L & F\end{array}$ 226 GCCAGTAACATTGGAAGCGGCCACTTTGTGGGACTGGCGGGGACCGGAGCAGCTGCAGGAATTGCCACTGGGGGC

$\begin{array}{llllllllllllllllllllllllllllllllllll}A & S & N & I & G & S & G & H & F & V & G & L & A & G & T & G & A & A & A & G & I & A & T & G & G & 100\end{array}$

301 TTTGAATGGAATGCCCTGATTTTGGTGGTTGTGCTGGGCTGGGTGTTTGTCCCCATTTACATTAAGGCGGGGGTG $\begin{array}{lllllllllllllllllllllllllll}F & E & \text { W } & \text { N } & A & \text { L } & \text { I } & \text { L } & \text { V } & \text { V } & \text { V } & \text { L } & G & \text { W } & \text { V } & \text { F } & \text { V } & \text { P } & \text { I } & \text { Y } & \text { I } & \text { K } & \text { A } & \text { G } & \text { V } & 125\end{array}$ 376 GTGACGATGCCAGAGTACCTGCGGAAGCGATTTGGAGGCCAGCGGATCCAGGTCTACCTGTCTGTCCTGTCCCTG $\begin{array}{llllllllllllllllllllllllllll}V & T & M & P & E & Y & L & R & K & R & F & G & G & Q & R & I & Q & V & Y & L & S & V & L & S & L & 150\end{array}$ 451 GTGCTGTACATTTTCACCAAGATCTCGGCAGACATCTTTTCTGGGGCCATATTCATCAACTTGGCCTTGGGCCTG $\begin{array}{llllllllllllllllllllllllllll}V & L & Y & I & F & T & K & I & S & A & D & I & F & S & G & A & I & F & I & N & L & A & L & G & L & 175\end{array}$ 526 GATCTCTACCTGGCCATCTTTATCTTATTGGCAATCACCGCCCTTTACACAATCACAGGGGGCCTGGCGGCTGTG

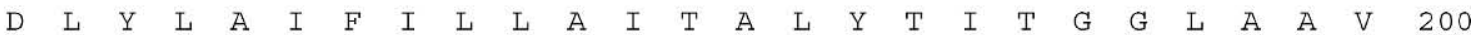

601 ATTCACACAGACACCTTGCAGACGGTGATTATGCTCCTGGGGTCTTTCATCCTGACTGGGTTTGCTTTCCACGAA

$\begin{array}{lllllllllllllllllllllllllllll}I & H & T & D & T & L & Q & T & V & I & M & L & L & G & S & F & I & L & T & G & F & A & F & H & E & 225\end{array}$ 676 GTGGGAGGGTATGGTGCCTTCGTGACAAAATACATGAATGCCATTCCAACTGTGACCTCTTATGGAAACACCACC $\begin{array}{llllllllllllllllllllllllllll}V & G & G & Y & G & A & F & V & T & K & Y & M & N & A & I & P & T & V & T & S & Y & G & N & T & T & 250\end{array}$ 751 ATCAAGGCAGAGTGCTACACCCCGAGGGCTGACTCCTTCCACATCTTCCGCGATCCTCTCAAGGGAGACCTGCCC

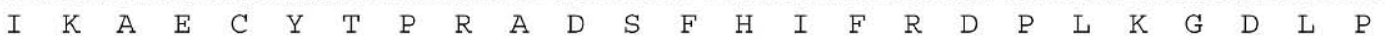
TGGCCTGGGCTCATCTTTGGGCTGACCATCATCTCCCTGTGGTACTGGTGCACAGACCAGGTCATTGTGCAGCGC

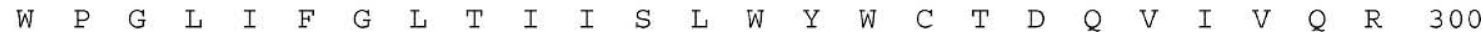
901 TGCCTCTCTGCCAAAAACATGTCCCACGTGAAGGCCGGCTGCATCATGTGTGGGTACCTGAAGCTGCTGCCCATG

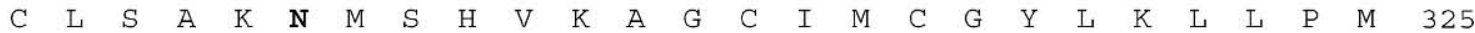
976 TTCCTCATGGTGATGCCAGGGATGATCAGCCGCATCCTGTTCACAGAGAAAGTTGCCTGCACCGTCCCCTCAGAG

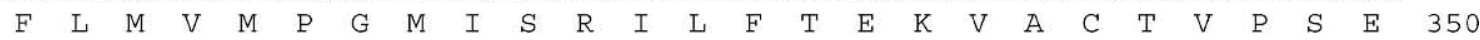

1051 TGTGAGAAATACTGCGGCACCAAGGTTGGCTGTACCAACATCGCCTACCCGACCTTGGTGGTGGAGCTCATGCCC

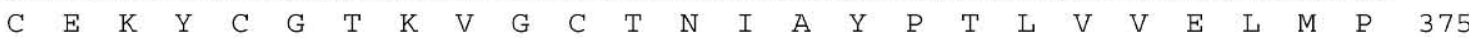

1126 AATGGACTGCGAGGCCTGATGCTGTCGGTCATGCTGGCCTCGCTCATGAGCTCGCTGACCTCCATCTTCAACAGT $\begin{array}{lllllllllllllllllllllllllllll}N & G & L & R & G & L & M & L & S & V & M & L & A & S & L & M & S & S & L & T & S & I & F & N & S & 400\end{array}$ 1201 GCCAGCACCCTCTTCACCATGGACATCTACACCAAGATCCGGAAGAAAGCCTCTGAGAAAGAGCTCATGATCGCA $\begin{array}{lllllllllllllllllllllllllllll}A & S & T & L & F & T & M & D & I & Y & T & K & I & R & K & K & A & S & E & K & E & L & M & I & A & 425\end{array}$ 1276 GGACGGTTGTTCATGCTGGTGCTGATCGGTGTCAGCATCGCCTGGGTGCCCATCGTGCAGTCAGCACAGAGTGGA $\begin{array}{lllllllllllllllllllllllllll}G & R & L & F & M & L & V & \text { L } & I & G & V & S & I & A & W & V & P & I & V & Q & S & A & Q & S & G & 450\end{array}$ 1351 CAGCTCTTCGACTACATCCAGTCCATCACCAGTTACTTGGGACCACCCATCGCAGCAGTCTTCCTGCTTGCTATT $\begin{array}{lllllllllllllllllllllllllll}Q & L & F & D & Y & I & Q & S & I & T & S & Y & L & G & P & P & I & A & A & V & F & L & L & A & I & 475\end{array}$ 1426 TTCTGCAAGAGAGTCAATGAGCCGGGAGCCTTTTGGGGATTGATCATAGGATTTTTGATCGGGGTGTCCCGTATG $\begin{array}{llllllllllllllllllllllllllll} & \mathrm{F} & \mathrm{C} & \mathrm{K} & \mathrm{R} & \mathrm{V} & \mathrm{N} & \mathrm{E} & \mathrm{P} & \mathrm{G} & \mathrm{A} & \mathrm{F} & \mathrm{W} & \mathrm{G} & \mathrm{L} & \mathrm{I} & \mathrm{I} & \mathrm{G} & \mathrm{F} & \mathrm{L} & \mathrm{I} & \mathrm{G} & \mathrm{V} & \mathrm{S} & \mathrm{R} & \mathrm{M} & 500\end{array}$ 1501 ATTACCGAGTTTGCCTATGGAACCGGGAGCTGCATGGAGCCCAGCAACTGTCCCACGATTATCTGTGGTGTGCAC $\begin{array}{llllllllllllllllllllllllllll}I & T & E & F & A & Y & G & T & G & S & C & M & E & P & S & N & C & P & T & I & I & C & G & V & H & 525\end{array}$ 1576 TACTTGTACTTTGCCATCATCCTCTTTGTCATTACTATCATCGTCATCCTGGCTGTCTCCCTCTTCACCAAGCCC $\begin{array}{lllllllllllllllllllllllllll}Y & L & Y & F & A & I & I & L & F & V & I & T & I & I & V & I & L & A & V & S & L & F & T & K & P & 550\end{array}$ 1651 ATTGCAGATGTGCATCTCTACCGCCTGTGCTGGAGCCTGCGTAACAGCAAAGAGGAGCGTATTGACCTGGATGCA

$\begin{array}{llllllllllllllllllllllllll}I & A & D & V & H & L & Y & R & L & C & W & S & L & R & N & S & K & E & E & R & I & D & L & D & A & 575\end{array}$ 1726 GAAGACGAGGACGTTCAAGATGCCCGGGAGGATGCCTTAGAAATAGACACAGAAGCTTCTGAGGAGAAAAAAGGA

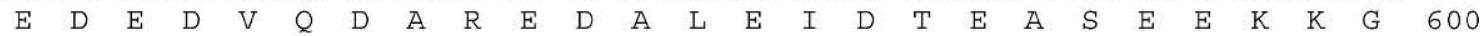
1801 TGCTTCAGGCGGGCCTATGACATGTTCTGTGGCCTGGACCAGCAGAAAGCCCCCAAAATGACGAAGGAAGAGGAG $\begin{array}{llllllllllllllllllllllllll}C & F & R & R & A & Y & D & M & F & C & G & L & D & Q & Q & K & A & P & K & M & T & K & E & E & E & 625\end{array}$ 1876 GCAGCCATGAAGCTGAAGATGACAGACACCTCGGAGAATCGTTTGTGGCGGATGGTAGTGAACATCAATGGCATC

$\begin{array}{lllllllllllllllllllllllllll}A & A & M & K & L & K & M & T & D & T & S & E & N & R & L & W & R & M & V & V & N & I & N & G & I & 650\end{array}$

1951 ATTCTGCTGGCCGTGGCTGTCTTCTGCCATGCCTACTTTGCCTGAgtcetgetctctgctgaggetgtgettgtg

$\begin{array}{llllllllllllllllll}I & \mathrm{~L} & \mathrm{~L} & \mathrm{~A} & \mathrm{~V} & \mathrm{~A} & \mathrm{~V} & \mathrm{~F} & \mathrm{C} & \mathrm{H} & \mathrm{A} & \mathrm{Y} & \mathrm{F} & \mathrm{A} & \text { * }\end{array}$

2026 gaggctggagtcttcactgtgtccetcttggccccattcagtggtgttgaagggataccagccagttgtaaact 2101 ctgccctgtcgataaatgtgtacatttgtaattacaggctagctgaagaaaaatcactgattcactgttaactta

2176 tatattcaaggccagtgtggtacagtcacctgtacatatctgagctgcagaagg

Figure 1. Full-length cDNA and deduced amino acid sequence of bovine SGLT1. In the cDNA sequence, lowercase letters represent the $5^{\prime}$ and $3^{\prime}$ untranslated regions, and uppercase letters represent the coding region. The predicted amino acid sequence is shown beneath the coding sequence. The predicted N-glycosylation sites are in bold face. The nucleotide sequence of bovine SGLT1 mRNA has been submitted to the GenBank nucleotide database with accession number AF508807. 
acgcggggagtcccactcgetcagagccggctctgctgcagg ATGGCTGTGGACAACTCCACCAGCGACGCCCATACCCCCGGGCGGCAGCTGACCGTGGTGGACATTGCCATCATC $\begin{array}{lllllllllllllllllllllllllll}M & A & V & D & N & S & T & S & D & A & H & T & P & G & R & Q & L & T & V & V & D & I & A & I & I\end{array}$

76 GCCGTGTATTTTGCCCTGAACGTGGCCGTGGGCATATGGTCCTCCTGCCGGGCCAGCAGGAACACAGTGAGAGGC

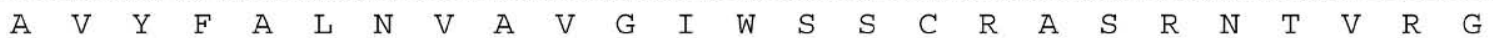

151 TACTTCCTGGCCGGCCGGGACATGACGTGGTGGCCGATCGGAGCCTCCCTTTTTGCCAGCAGTGAGGGTTCGGGT

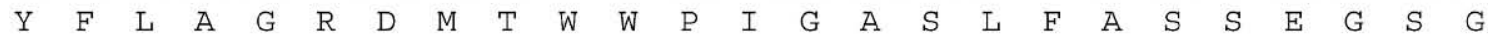

226 СTCTTCATTGGGCTGGCGGGCTCGGGAGCGGCTGGAGGCTTGGCGGTGGCTGGCTTCGAGTGGAATGCTACCTAT

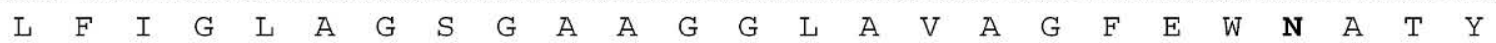
GTGCTGCTGGCCCTGGCATGGGTGTTTGTGCCCATCTACCTCTCCTCGGAGATCGTCACCATGCCTGAATACATG $\begin{array}{lllllllllllllllllllllllllllllllll}V & L & L & A & L & A & W & V & F & V & P & I & Y & L & S & S & E & I & V & T & M & P & E & Y & M\end{array}$ 376 CAGAAGCGCTACGGGGGCCAGCGGATTCGCATGTACCTGTCTGTCCTGTCGCTGCTGCTGTCCGTCTTCACCAAG $\begin{array}{lllllllllllllllllllllllllll}Q & K & R & Y & G & G & Q & R & I & R & M & Y & L & S & V & L & S & L & L & L & S & V & F & T & K\end{array}$

451 ATATCGATTGACCTGTATGCGGGGGCCCTGTTCGTGCACATCTGCCTGGGCTGGAACTTCTACCTGTCCACGGTC

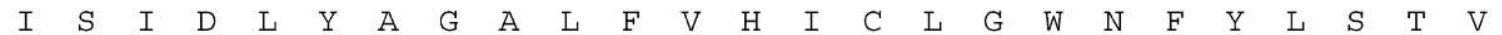
526 ATCATGCTCGCCATCACGGCCCTTTACACCATCGCAGGGGGCCTGACTGCTGTCATCTACACAGACGCCCTGCAG

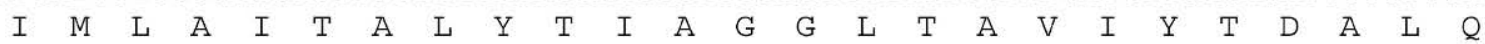
ACGCTCGTCATGGTGGCGGGGGCCGTGATCCTGACGATCAAAGCTTTCGAGCAGATTGGCGGGTACGAGCAGCTG

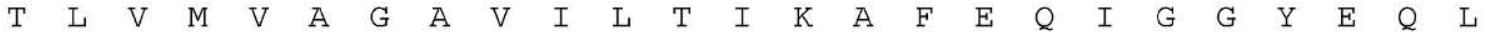
676 GCAGAGGCCTACGCCCAGGCCGTCCCATCCAGGACCATCTCCAATACCACGTGCCACGTGCCCCGGGCAGACGCC

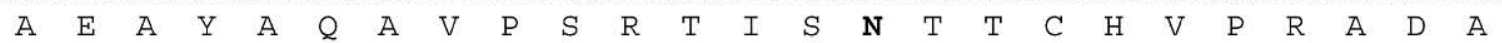
ATGCATATGTTCCGAGACCCCTACACCGCGGACCTCCCGTGGACTGGGATGACCTTCGGCCTGACCATCATGGCC $\begin{array}{lllllllllllllllllllllllllll}M & H & M & F & R & D & P & Y & T & A & D & L & P & W & T & G & M & T & F & G & L & T & I & M & A\end{array}$ GCCTGGTACTGGTGCACTGACCAGGTCATCGTGCAGCGGTCACTGTCTGCCCGGGACCTGAACCACGCCAAGGGG

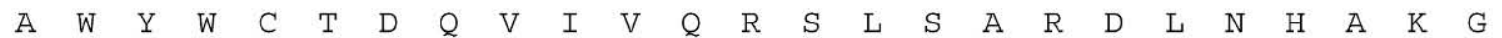
GGCTCCATCCTGGCCAGCTATCTCAAGATGCTGCCCATGGGCCTGATGGTCATGCCGGGCATGATCAGCCGCGTG $\begin{array}{llllllllllllllllllllllllll}G & S & I & L & A & S & Y & L & K & M & L & P & M & G & L & M & V & M & P & G & M & I & S & R & V\end{array}$ CTGTTCCCAGATGACGTGGGCTGTGTGGTGCCAGCCGAGTGCCTGCGGGCCTGCGGGGCTGAGATCGGCTGCTCC

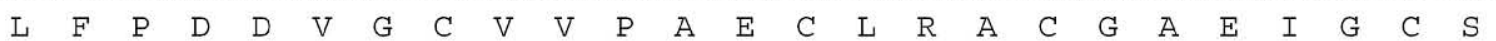
1051 AACATCGCCTACCCCAAGCTGGTCATGGAGCTGATGCCCACGGGTCTGCGGGGGCTGATGGTCGCTGTGATGATG $\begin{array}{llllllllllllllllllllllllll}\mathrm{N} & I & A & Y & P & K & L & V & M & E & L & M & P & T & G & L & R & G & L & M & V & A & V & M & M\end{array}$ 1126 GCTGCGCTCATGTCCTCGCTGACCTCCATCTTCAATAGCAGCAGCACCCTCTTCACCATGGACATCTGGAGGCGG

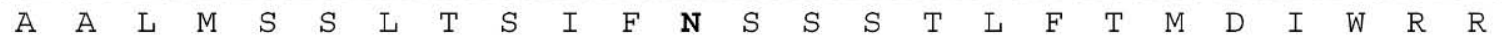
1201 CTGCGGCCCCGTGCGGGCGAGCGGGAGCTGCTGCTGGTGGGACGGCTGGTCATCGTGGTGCTCGTCGGCGTGAGT $\begin{array}{llllllllllllllllllllllllllllll}L & R & P & R & A & G & E & R & E & L & L & L & V & G & R & L & V & I & V & V & L & V & G & V & S\end{array}$ 1276 GTGGCTTGGATCCCCGTCCTGCAGGGCTCCAATGGCGGGCAGCTCTTCATCTACATGCAGTCGGTGACCAGCTCC

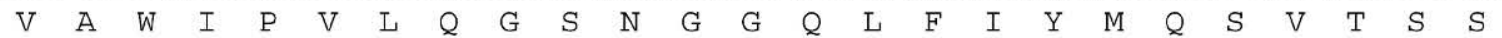
1351 CTGGCCCCACCGGTGACCGCAGTCTTCGTTTTGGGCATCTTCTGGCGGCGGGCCAACGAGCAGGGAGCCTTCTGG

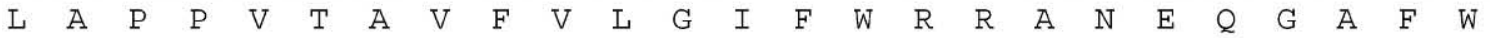
1426 GGCCTGATGGCGGGGCTGGCAGTGGGTGCCACGAGGCTGGTGCTGGAGTTCTTGCACCCAGCCCCGCCCTGCGGC $\begin{array}{llllllllllllllllllllllllll}G & L & M & A & G & L & A & V & G & A & T & R & L & V & L & E & F & L & H & P & A & P & P & C & G\end{array}$ 1501 CACCCTGACACGCGGCCCCCCATCCTCCATGGCGTCCACTACTTGCACTTCGCCGTGGCCCTCTTCCTGCTCAGC

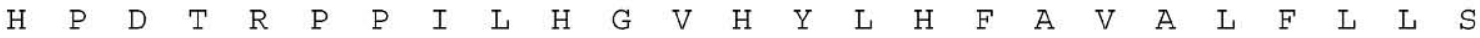
1576 GGGGCTGTGGTGGTCGCTGGGAGCCTGCTGACACCACACCCCCAGGGCGTCCAGATCCAGAGCCTGACCTGGTGG

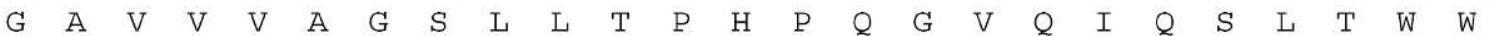

1651 ACTCTGGCTCAGGACCTGCCCTTGGGAGTGAAAACAGGTGATGGCCGAGCATCCCAGAGACACGCCTTCTGGGCT $\begin{array}{llllllllllllllllllllllllll}T & \mathrm{~L} & \mathrm{~A} & \mathrm{Q} & \mathrm{D} & \mathrm{L} & \mathrm{P} & \mathrm{L} & \mathrm{G} & \mathrm{V} & \mathrm{K} & \mathrm{T} & \mathrm{G} & \mathrm{D} & \mathrm{G} & \mathrm{R} & \mathrm{A} & \mathrm{S} & \mathrm{Q} & \mathrm{R} & \mathrm{H} & \mathrm{A} & \mathrm{F} & \mathrm{W} & \mathrm{A}\end{array}$ 1726 CGTGTCTGCGGTGTCAACGCCATCCTCCTCATGTGCGTCAACATCTTCTTTTACACCTACTTCGCCTGACgCtgC $\begin{array}{llllllllllllllllllllllll}R & \mathrm{~V} & \mathrm{C} & \mathrm{G} & \mathrm{V} & \mathrm{N} & \mathrm{A} & \mathrm{I} & \mathrm{L} & \mathrm{L} & \mathrm{M} & \mathrm{C} & \mathrm{V} & \mathrm{N} & \mathrm{I} & \mathrm{F} & \mathrm{F} & \mathrm{Y} & \mathrm{T} & \mathrm{Y} & \mathrm{F} & \mathrm{A} & \text { * }\end{array}$

1801 ccccagagtaccccttccccgtcaagaacaccgagggccagaggagggcagcgccccttgggccacagggcagg 1876 ccagtgcccagcgactggccaagccagccaagtaggagccctgaaaattagggaggaaatgggaaaaaaagaat 1951 gtctgatactttaaaatagtagttgtaattggaaagaaaatgagatttctcacgggcaaaaaaaaaaagaaaa 2026 aaaaaaaaaaa

Figure 2. Full-length cDNA and deduced amino acid sequence of bovine SGLT5. In the cDNA sequence, lowercase letters represent the $5^{\prime}$ and $3^{\prime}$ untranslated regions, and uppercase letters represent the coding region. The predicted amino acid sequence is shown beneath the coding sequence. The predicted N-glycosylation sites are in bold face. The nucleotide sequence of bovine SGLT5 mRNA has been submitted to the GenBank nucleotide database with accession number AY514442. 


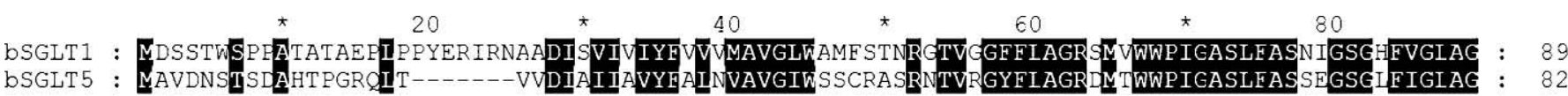

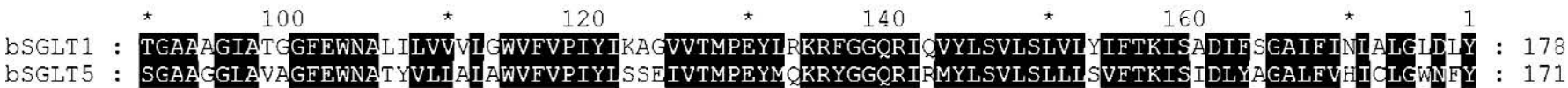

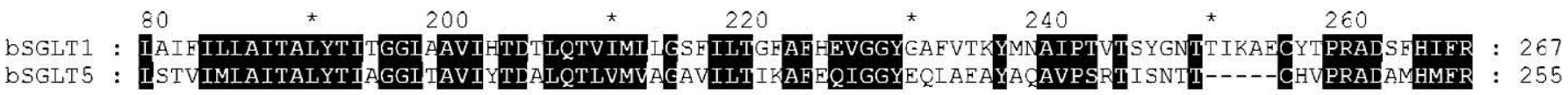

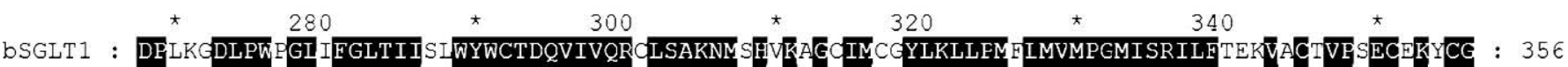
bSGLT5 : DPYTADLPWTGMTEGLTINAAWYWCTDQVIVQRSLSARDINEAKGGSIIASYLKMLFNGLMVMPGMISRVLFPDIVGOVVHAEOLACCG : 344

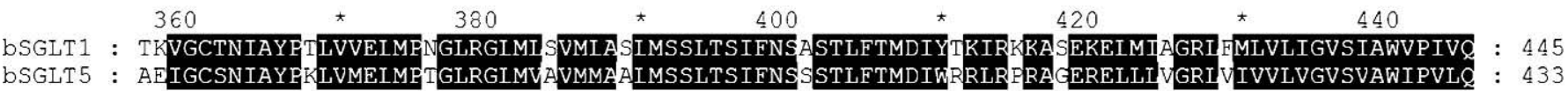

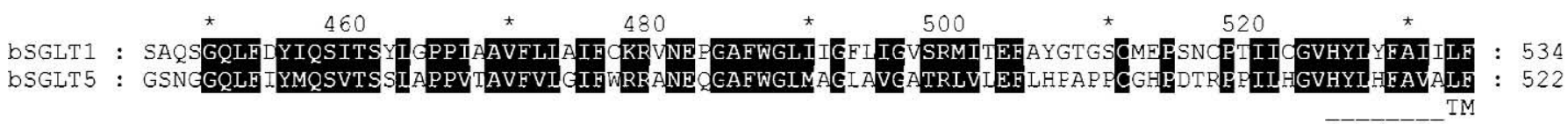

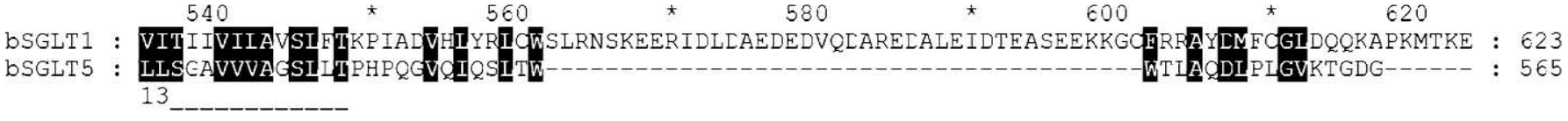

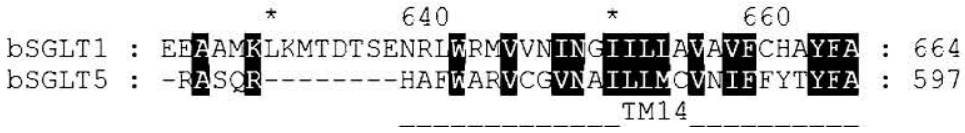

Figure 3. Sequence alignment of the deduced amino acid sequences of bovine SGLT1 and SGLT5. The alignment was performed with the CLUSTAL W program. Residues with a black background represent absolutely conserved amino acids. The positions of presumed membrane-spanning helices (TM 13 and 14) in SGLT1 are shown by the numbered dashed lines below the sequence alignments.

differences between the 2 transporters. The major differences are in the cytoplasmic loop between TM 13 and 14, which is much longer in SGLT1. There is little sequence homology in this loop (Figure 3).

The sequences of bSGLT1 and bSGLT5 contain several conserved sodium:solute symporter family signatures that are characteristic of the sodium:solute symporter family. The sodium:solute symporter family signature 1 (NA_SOLUT_SYMP_1; accession no.PS00456) is located between amino acids 174 and 199 (GldLylaIFILLAItaIYTitGGLaA) in TM 5 of bSGLT1. The sodium:solute symporter family signature 2 (NA_SOLUT_SYMP_2; PS00457) is located between amino acids 474 and 494 (AIfckRvnepGAfwGLIIGfL) between TM 11 and loop 12 of bSGLT1 and between amino acids 462 and 482 (GIfwrRaneqGAfwGLMAGlA) of bSGLT5. In addition, the sequences between amino acids 25 and 506 in bSGLT1 and between amino acids 18 and 494 in bSGLT5 match with the sodium:solute symporter family profile (PS50283).

Scan-Prosite analysis predicted 2 potential N-glycosylation sites positioned at amino acids 248 to 251 (NTTI) and 306 to 309 (NMSH) of bSGLT1, and 4 sites at amino acids 5 to 8 (NSTS), 97 to 100 (NATY), 240 to 243 (NTTC), and 387 to 390 (NSSS) of bSGLT5 (Figures 1 and 2). In addition, numerous cAMP- and cGMP-dependent protein kinase, protein kinase $\mathrm{C}$, tyrosine kinase, and casein kinase II phosphorylation sites are also predicted with a high probability of occurrence, although the functional significance of these sites remains to be investigated. It has been shown that when rabbit SGLT1 is expressed in oocytes, the maximum rate of $\mathrm{Na}^{+} /$glucose cotransport of the oocytes is increased by $30 \%$ by the activation of protein kinase A, but decreased by $60 \%$ by the activation of protein kinase $\mathrm{C}$, and the effects depend on the sequence of the cotransporter being expressed (Wright et 


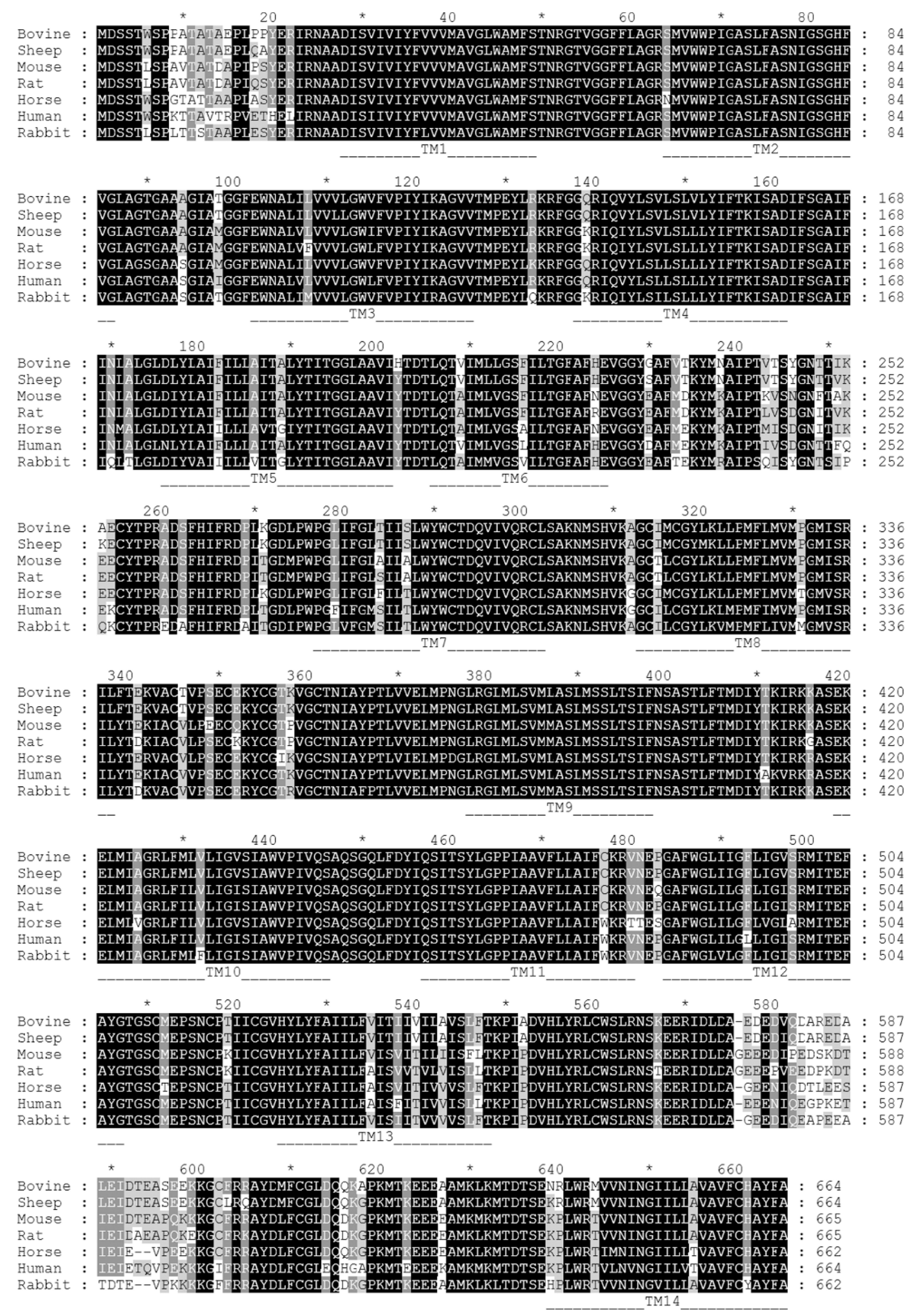

Figure 4. Multiple sequence alignment of the deduced amino acid sequence of bovine SGLT1 with the sequences of sheep (GenBank accession number X82411), mouse (AF208031), rat (D16101), horse (AJ292081), human (M24847), and rabbit (X55355). The alignment was performed with the CLUSTAL W program (open gap cost 10). Residues with a black background represent absolutely conserved amino acids; gray shading indicates 4 or more conserved residues at that position. Positions of presumed membrane-spanning helices (TM) are given by the numbered, dashed lines below the sequence alignments. 

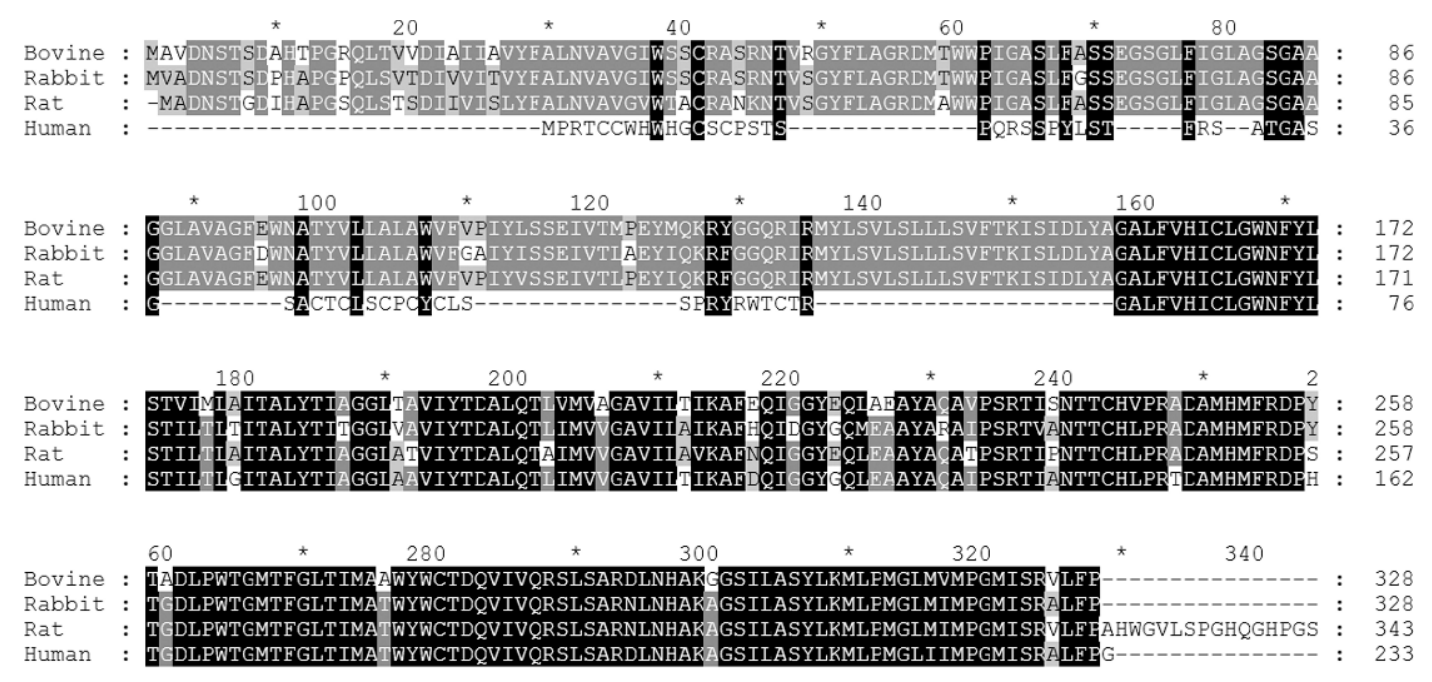

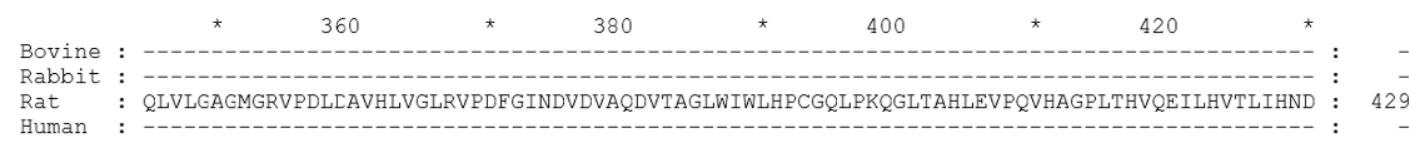

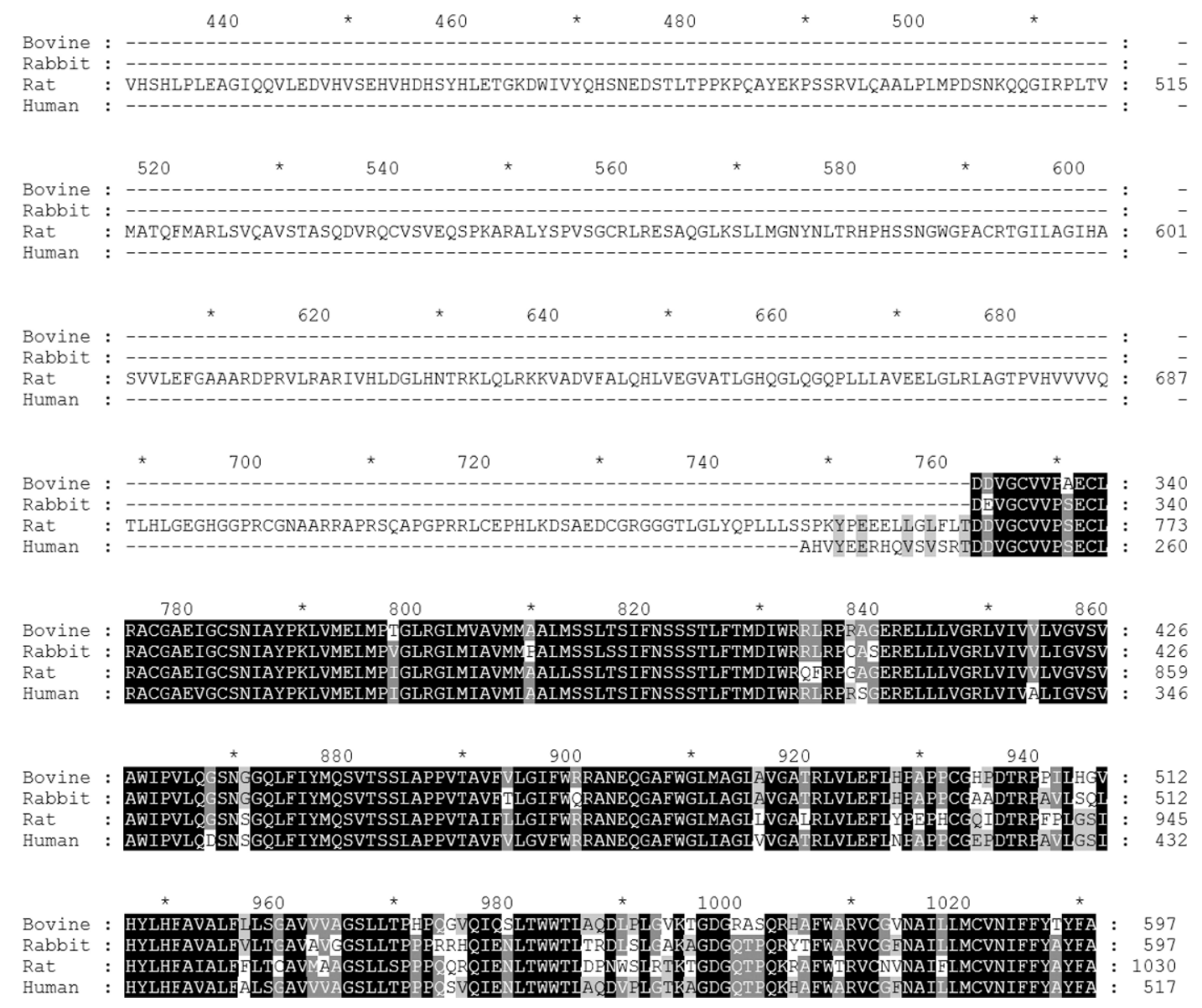

Figure 5. Multiple sequence alignment of the deduced amino acid sequence of bovine SGLT5 with the sequences of rabbit (GenBank accession number U08813), rat (XM_220540), and human (AK057946). The alignment was performed with the CLUSTAL W program (open gap cost 10). Residues with a black background represent absolutely conserved amino acids; gray shading indicates 2 or more conserved residues at that position. 
A.

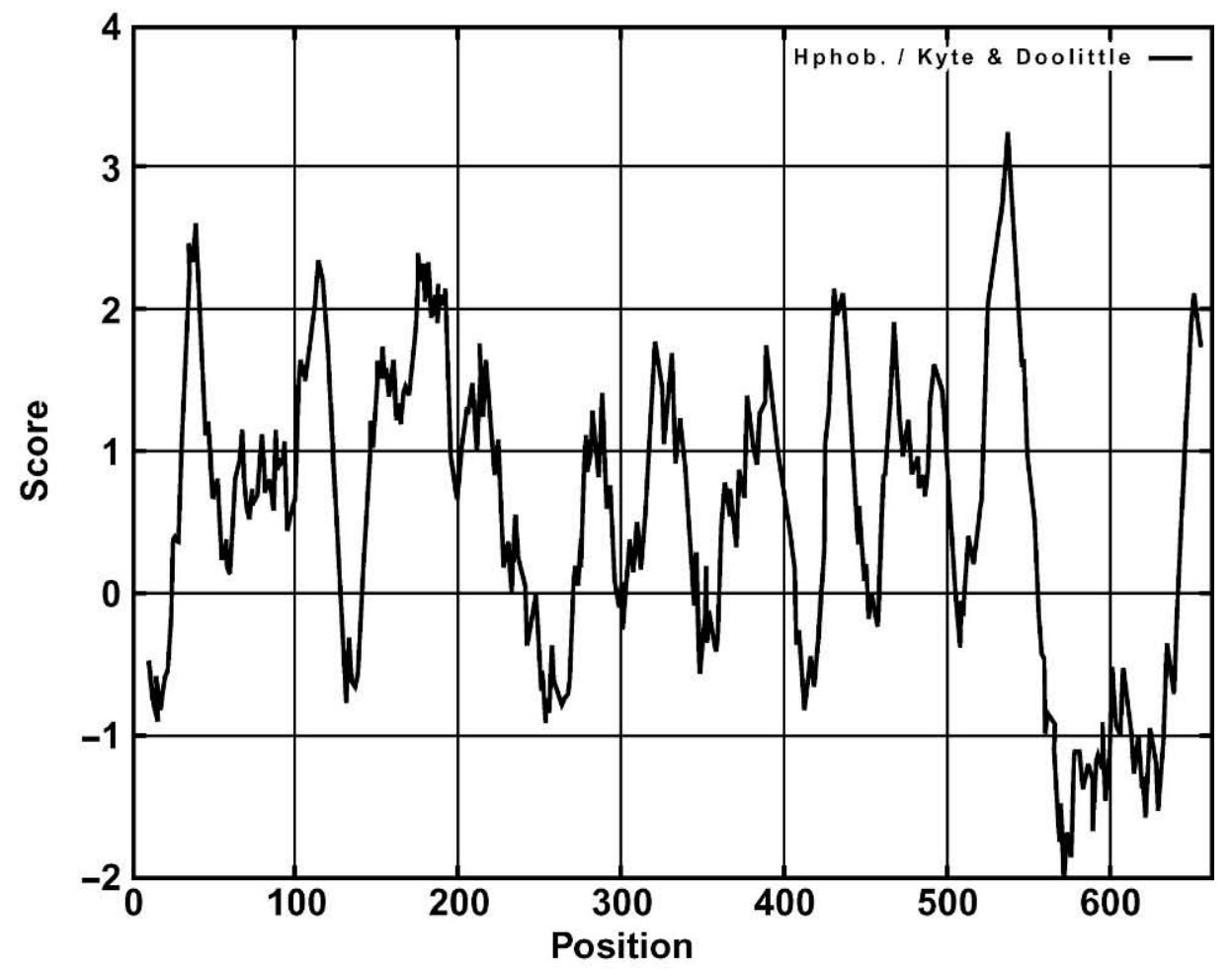

B.

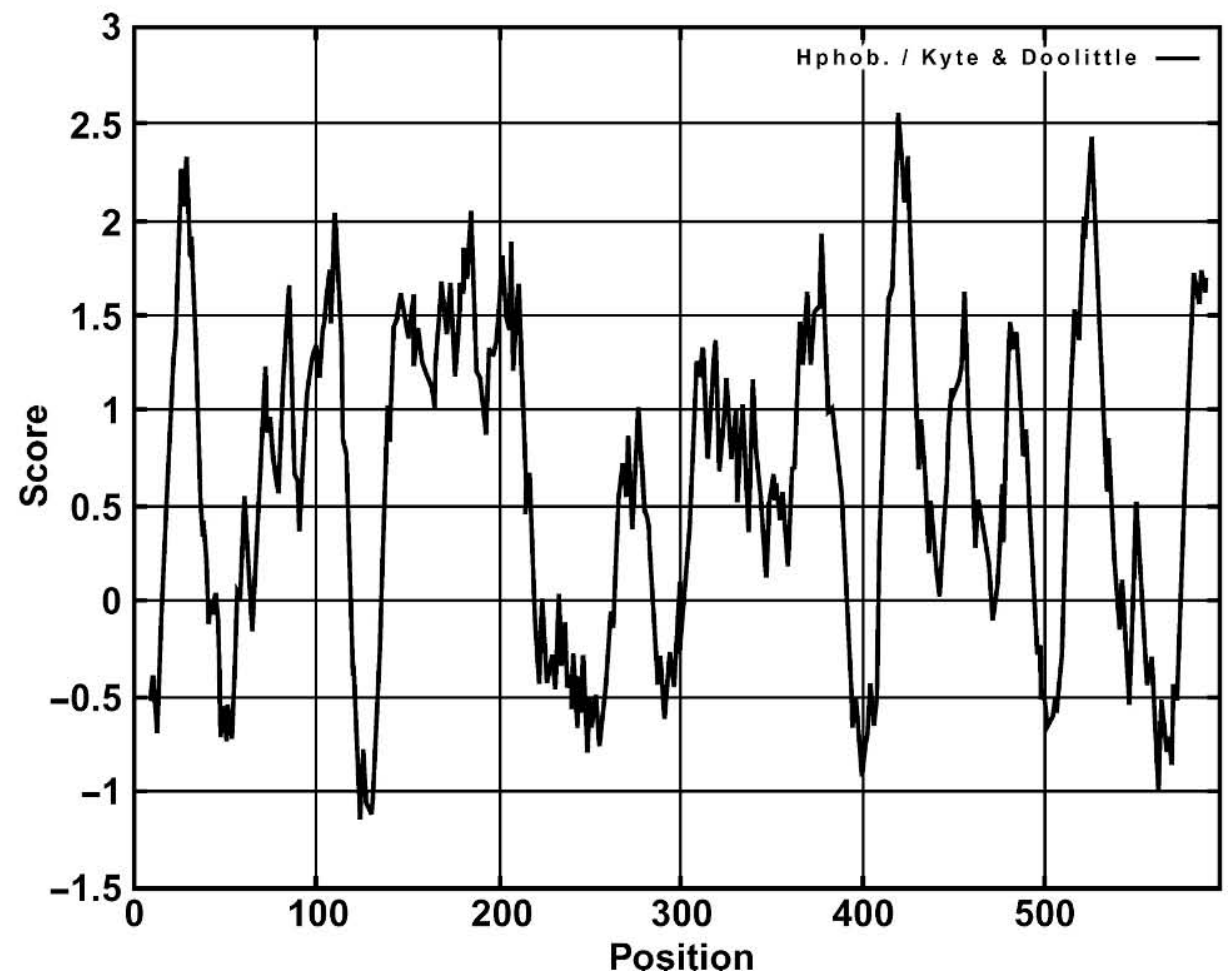

Figure 6. Hydropathy plots of bovine SGLT1 (A) and SGLT5 (B). The hydropathy plots were derived according to the algorithm of Kyte and Doolittle (1982), using a 19-AA window. 

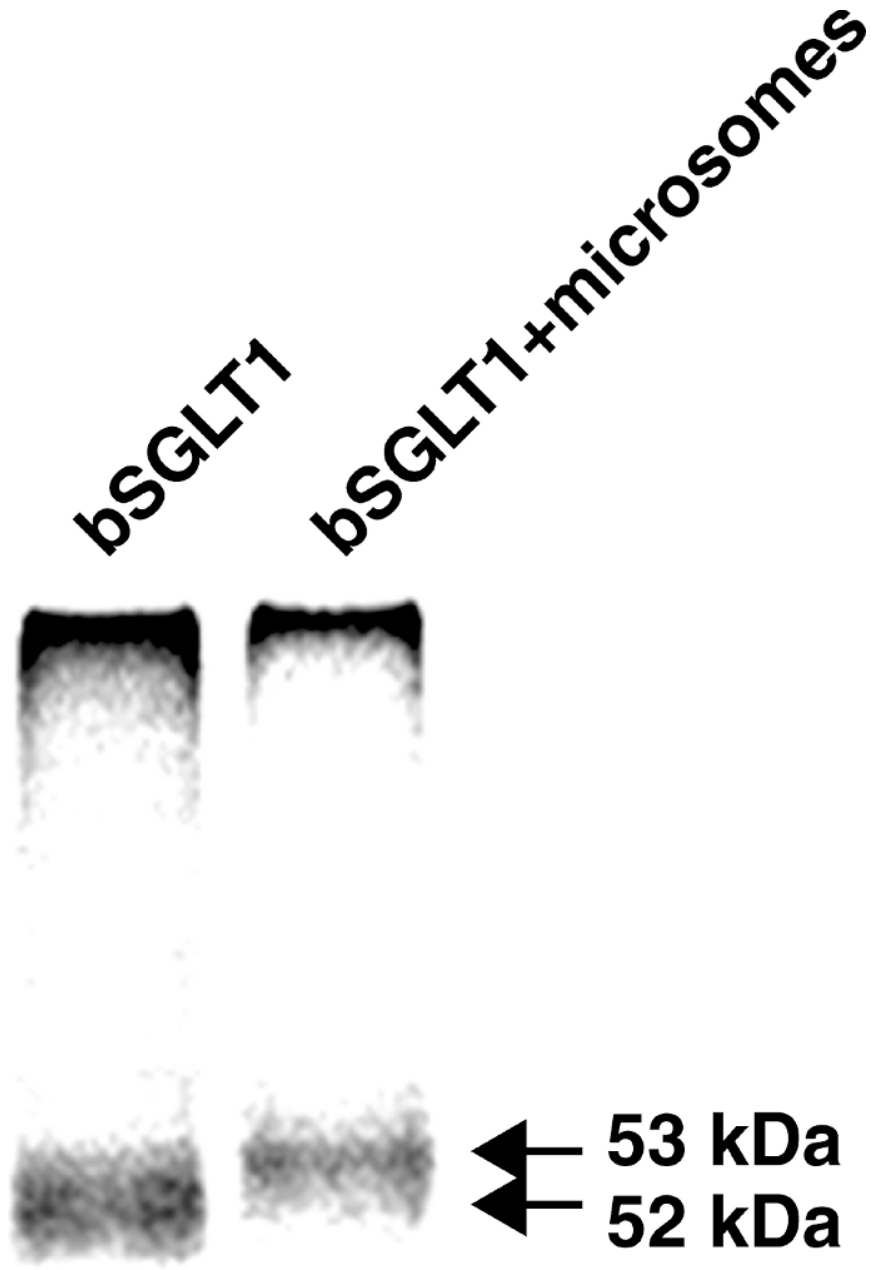

Figure 7. In vitro transcription and translation of bovine SGLT1 cDNA with or without addition of canine microsomal membranes in the reaction. The positions of translation products are indicated.

al., 1997). Phosphorylation of SGLT1 regulates its transport activity by stimulating rapid insertion into the plasma membrane (Hirsch et al., 1996). However, at least the effect of protein kinase $\mathrm{A}$ is independent of the presence or absence of consensus phosphorylation sites on SGLT1 (Wright et al., 1997), suggesting that the effect of the protein kinases is indirect.

\section{In Vitro Transcription and Translation of bSGLT1 cDNA and Glycosylation of bSGLT1}

Coupled in vitro transcription and translation were used to characterize the bSGLT1 gene product (Figure 7). The translation product migrated at an apparent molecular weight of $52 \mathrm{kDa}$, which differed from the sizes we reported in our Western blot analysis of SGLT1 in bovine tissues (Zhao et al., 1998, 1999), and was considerably smaller than the $73 \mathrm{kDa}$ predicted from the se- quence of the open reading frame. This inconsistency was also observed for bGLUT1, bGLUT8, and bSGLT2 (Zhao et al., 2004, and unpublished data) and may be due to different initiation sites used in translation. For example, the calculated protein size would be $51 \mathrm{kDa}$ if the translation of bSGLT1 starts at methionine at the 211th amino acid position of full length sequence, and would retain 2 potential glycosylation sites. On addition of canine microsomal membranes to the translation reactions, the apparent size increased to $53 \mathrm{kDa}$, suggesting that the protein was glycosylated. This is consistent with the 2 predicted N-glycosylation sites in bSGLT1 (see above). However, the functional significance of the glycosylation of bSGLT1 is not known because it has been shown that glycosylation is not required for the activity of human SGLT1 (Wright and Turk, 2004).

It is worth noting that all glucose transporters strongly aggregate even under standard denaturing conditions, making it necessary to add $3 M$ urea to the gel and sample buffer to reduce protein aggregation.

\section{Distribution of SGLT1 and SGLT5 mRNA in Bovine Tissues}

Expression of SGLT1 and SGLT5 was analyzed by RTPCR in the mammary gland, liver, kidney, lung, spleen, intestine, and skeletal muscle of 2 lactating cows, and in testicular tissue of 1 bull (Figure 8) using specific primers derived from each cDNA. The mRNA for SGLT1 was found most abundant in bovine intestine, at intermediate levels in bovine kidney, and at lower levels in bovine mammary gland, liver, and lung. No SGLT1 mRNA was detected in bovine spleen, skeletal muscle, and testes. The relative expression levels of SGLT1 mRNA in bovine intestine, kidney, mammary gland, and liver are consistent with our previous results from Northern blot analysis using a human cDNA probe (Zhao et al., 1998, 1999). Our current findings confirm these results and extend the range of tissues examined.

Consistent with the distribution reported in rabbit (Pajor, 1994), SGLT5 mRNA is predominantly expressed in bovine kidney. It is also expressed at very low levels in bovine testes, skeletal muscle, and spleen, and is essentially undetectable in bovine mammary gland, liver, lung, and small intestine. In addition, multiple PCR products of bSGLT5 with different sizes were amplified in various tissues (Figure 8), indicating possible presence of multiple different splicing transcripts of SGLT5 in these tissues. Although 2 PCR bands were amplified in kidney for bSGLT5, we could only clone 1 full transcript. This may be due to very low level of the alternative transcripts and high GC-rich property of the sequences to be amplified. 


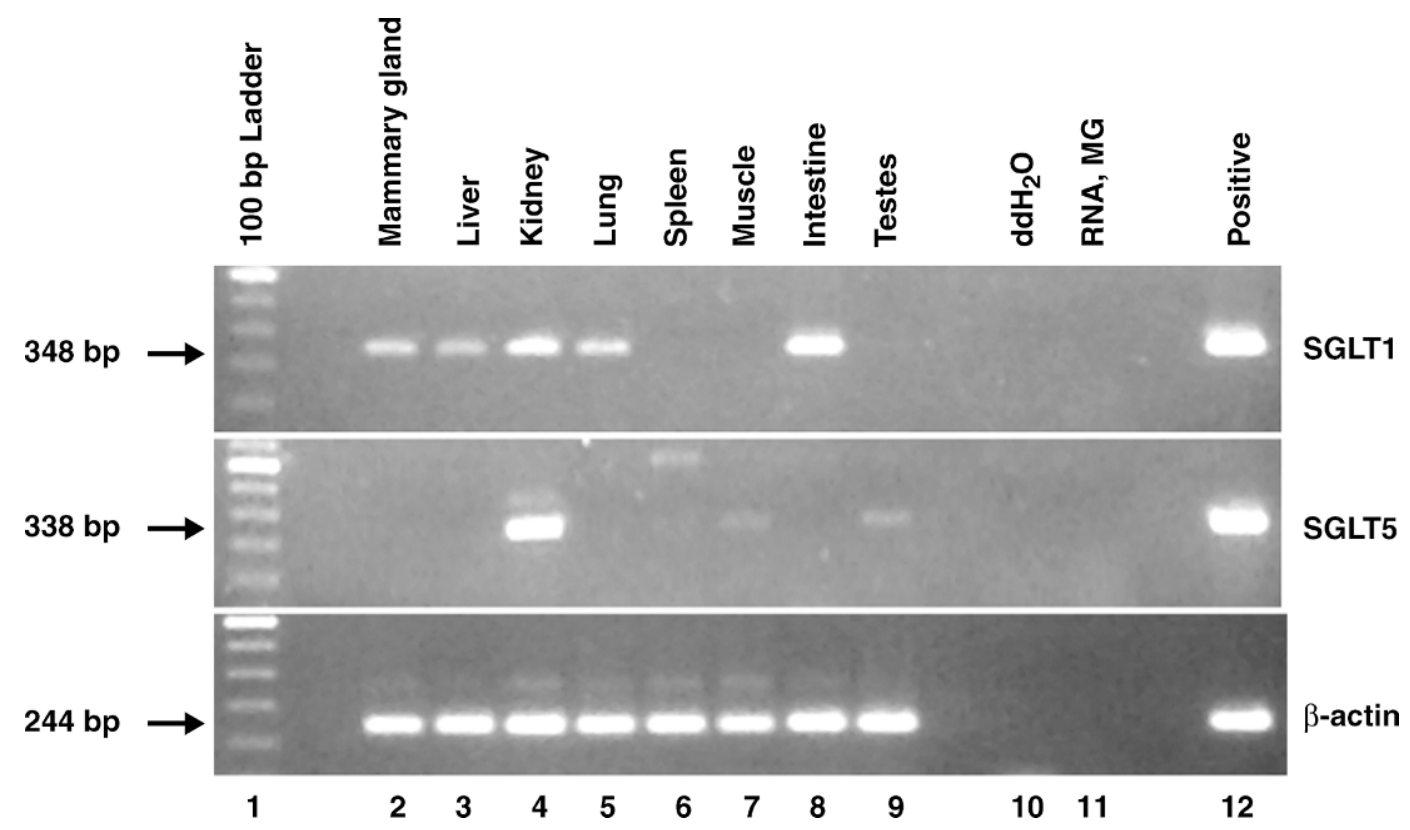

Figure 8. Tissue distribution of SGLT1 and SGLT5 mRNA in bovine by reverse transcription-PCR. The first-strand cDNA were synthesized from $5 \mu \mathrm{g}$ of total RNA (pooled from 2 lactating cows) isolated from the mammary gland (lane 2), liver (lane 3), kidney (lane 4), lung (lane 5), spleen (lane 6), skeletal muscle (lane 7), and small intestinal mucosa (lane 8), or from $5 \mu \mathrm{g}$ of total RNA isolated from testes (lane 9) of one bull. The cDNA, along with $\mathrm{ddH}_{2} \mathrm{O}$, RNA from the mammary gland (MG; lanes 10 and 11, negative controls) and $1 \mathrm{ng}$ of bSGLT1/pCRBlunt II-TOPO, bSGLT5/pCR4-TOPO plasmid DNA or pTRI- $\beta$-Actin-Mouse Antisense control template (lane 12, positive controls) were amplified using primers for bSGLT1 (upper panel), bSGLT5 (middle panel), or $\beta$-actin (bottom panel). The sizes of reverse transcriptionPCR products are indicated by arrows.

It is believed that SGLT1 plays a major role in intestinal uptake of dietary glucose and galactose because SGLT1 missense mutation is linked to hereditary glucose/galactose malabsorption characterized by potentially fatal diarrhea in humans (Wright et al., 2002), and is only considered to play a minor role in the reabsorption of glucose from the glomerular filtrate in kidney. The expression pattern of SGLT1 mRNA in bovine intestine and kidney indicates that SGLT1 may have similar roles in ruminants. The physiological significance of SGLT1 expression in the mammary gland, liver, and lung is uncertain. The expression of SGLT1 protein was confirmed in bovine mammary gland (Zhao et al., 1998, 1999). Because members of the sodium/glucose cotransporter family play multifunctional roles, such as functioning as uniporters, urea and water channels, and urea and water cotransporters (Wright and Turk, 2004), and they have multiple cotransported substrates (sugars, inositol, proline, pantothenate, iodide, urea, and undetermined solutes), it is a challenge to determine the physiological role(s) of these genes in individual tissues.

The functions of SGLT5 are completely unknown. The predominant expression of SGLT5 in kidney suggests that it may play a role in renal function.

\section{Developmental Regulation of SGLT1 mRNA Expression in Bovine Mammary Gland}

Because SGLT1 is expressed in bovine mammary gland, we wanted to determine whether its expression is developmentally regulated. We used quantitative RTPCR to analyze the SGLT1 mRNA levels in mammary tissues taken by biopsy from 12 cows on $d-40,-20,-7$, and +7 relative to calving (Figure 9). Similar to GLUT1 and GLUT8 (Zhao et al., 2004), expression of SGLT1 in bovine mammary gland is developmentally regulated during pregnancy and lactation. SGLT1 mRNA levels in bovine mammary gland increased more than 4 -fold $(P<$ 0.01 ) from $d-40$ to $d+7$ relative to parturition, compared with 10-fold and more than 100-fold increases for GLUT8 and GLUT1, respectively (Zhao et al., 2004).

Developmental regulation of SGLT1 in bovine mammary gland suggests that it may play a role in supporting lactation and implies that lactogenic hormones may regulate its expression. It will be important to determine the cellular and subcellular localization of SGLT1 in mammary tissue to gain insight into its function. For example, localization in Golgi membranes of mammary epithelial cells may indicate a role in uptake of glucose from cytosol to Golgi apparatus for lactose synthesis. 


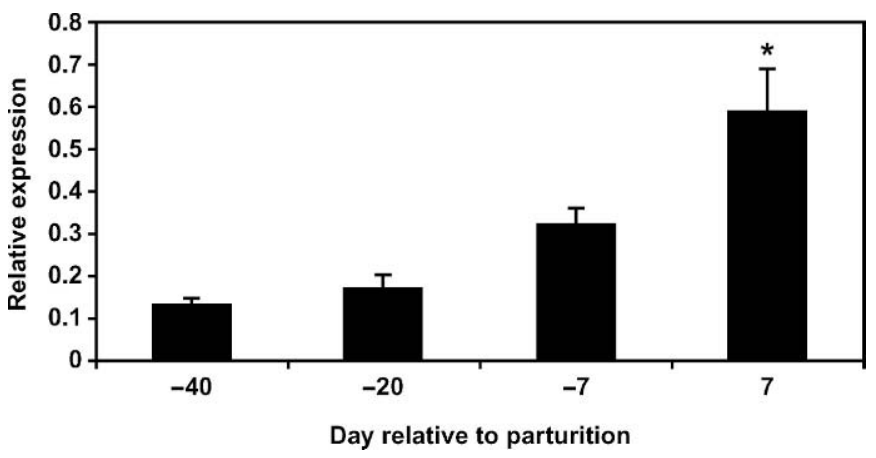

Figure 9. Developmental regulation of SGLT1 mRNA expression in the bovine mammary gland during pregnancy and lactation by quantitative reverse transcription-PCR analysis. The relative expression of bSGLT1 was normalized to $\beta$-actin levels. Data shown are means \pm SEM $(\mathrm{n}=12)$. The overall effect of developmental stage was significant $(P<0.01)$. Asterisks indicate significant differences relative to the previous time point $(P \leq 0.05)$.

Localization in apical membranes may suggest a role in transporting glucose between the alveolar lumen and epithelial cells. SGLT1 may also localize in the basolateral membranes where it could play a role in glucose uptake from extracellular fluid. However, because there is a large concentration gradient across the basolateral membranes of the cells, with a much higher concentration of glucose in the extracellular fluid (Faulkner et al., 1981), it is not clear why an active transporter would be involved in this process. The subcellular localization of SGLT1 in the bovine mammary gland is presently hampered by the lack of specific antibodies against this protein. The anti-SGLT1 antibodies used in the earlier studies are now known to cross-react with other SGLT isoforms.

\section{CONCLUSIONS}

In this paper, we report the cDNA and the deduced amino acid sequences of bovine SGLT1 and SGLT5 and their expression in bovine tissues. The expression and developmental regulation of SGLT1 in the mammary gland suggests that it may have a physiological role in milk synthesis. Our data lay the foundation for future studies aimed at unraveling the functional roles of sodium/glucose cotransporters in supporting milk production and maintaining glucose homeostasis during lactation.

\section{ACKNOWLEDGMENTS}

The work was supported by the United States Department of Agriculture Hatch grants VT-AS-00808 and VTAS-00915 (to FQZ) and by the United States-Israel Bina- tional Agricultural Research and Development Fund (BARD) US-3201-01R (to TBM).

\section{REFERENCES}

Farr, V. C., K. Stelwagen, L. R. Cate, A. J. Molenaar, T. B. McFadden, and S. R. Davis. 1996. An improved method for the routine biopsy of bovine mammary tissue. J. Dairy Sci. 79:543-549.

Faulkner, A., N. Chaiyabutr, M. Peaker, D. T. Carrick, and N. J. Kuhn. 1981. Metabolic significance of milk glucose. J. Dairy Res. 48:51-56.

Hirsch, J. R., D. D. Loo, and E. M. Wright. 1996. Regulation of $\mathrm{Na}^{+} /$ glucose cotransporter expression by protein kinases in Xenopus laevis oocytes. J. Biol. Chem. 271:14740-14746.

Jacobson, A. 1987. Purification and fractionation of Poly(A) ${ }^{+}$RNA. Meth. Enzymol. 152:254-261.

Kanai, Y., W. S. Lee, G. You, D. Brown, and M. A. Hediger. 1994. The human kidney low affinity $\mathrm{Na}^{+}$/glucose cotransporter SGLT2. Delineation of the major renal reabsorptive mechanism for D-glucose. J. Clin. Invest. 93:397-404.

Kong, C. T., S. F. Yet, and J. E. Lever. 1993. Cloning and expression of a mammalian $\mathrm{Na}^{+} /$amino acid cotransporter with sequence similarity to $\mathrm{Na}^{+}$/glucose cotransporters. J. Biol. Chem. 268:1509-1512.

Kyte, J., and R. F. Doolittle. 1982. A simple method for displaying the hydropathic character of a protein. J. Mol. Biol. 157:105-132.

Mackenzie, B., M. Panayotova-Heiermann, D. D. Loo, J. E. Lever, and E. M. Wright. 1994. SAAT1 is a low affinity $\mathrm{Na}^{+} /$glucose cotransporter and not an amino acid transporter. A reinterpretation. J. Biol. Chem. 269:22488-22491.

Pajor, A. M. 1994. Sequence of a putative transporter from rabbit kidney related to the $\mathrm{Na}^{+} /$glucose cotransporter gene family. Biochim. Biophys. Acta 1194:349-351.

Thompson, J. D., D. G. Higgins, and T. J. Gibson. 1994. CLUSTAL W: improving the sensitivity of progressive multiple sequence alignment through sequence weighting, positions-specific gap penalties and weight matrix choice. Nucleic Acids Res. 22:4673-4680.

Turk, E., and E. M. Wright. 1997. Membrane topology motifs in the SGLT cotransporter family. J. Membr. Biol. 159:1-20.

Xiao, C., and J. P. Cant. 2003. Glucose transporter in bovine mammary epithelial cells is an asymmetric carrier that exhibits cooperativity and trans-stimulation. Am. J. Physiol., Cell Physiol. 285:C1226C1234.

Wells, R. G., A. M. Pajor, Y. Kanai, E. Turk, E. M. Wright, and M. A. Hediger. 1992. Cloning of a human kidney cDNA with similarity to the sodium-glucose cotransporter. Am. J. Physiol. 263:F459-F465.

Wood, I. S., and P. Trayhurn. 2003. Glucose transporters (GLUT and SGLT): Expanded families of sugar transport proteins. Br. J. Nutr. 89:3-9.

Wright, E. M. 1993. The intestinal $\mathrm{Na}^{+} /$glucose cotransporter. Annu. Rev. Physiol. 55:575-589.

Wright, E. M., J. R. Hirsch, D. D. Loo, and G. A. Zampighi. 1997. Regulation of $\mathrm{Na}^{+}$/glucose cotransporters. J. Exp. Biol. 200:287293.

Wright, E. M., and E. Turk. 2004. The sodium/glucose cotransport family SLC5. Pflugers Arch. 447:510-518.

Wright, E. M., E. Turk, and M. G. Martin. 2002. Molecular basis for glucose-galactose malabsorption. Cell Biochem. Biophys. 36:115121.

Zhao, F.-Q., P. Miller, E. H. Wall, Y.-C. Zheng, B. Dong, M. C. Neville, and T. B. McFadden. 2004. Bovine glucose transporter GLUT8. Cloning, expression, and developmental regulation in mammary gland. Biochim. Biophys. Acta 1680:103-113.

Zhao, F.-Q., E. R. Okine, C. I. Cheeseman, S. P. Shirazi-Beechey, and J. J. Kennelly. 1998. Gene expression of $\mathrm{Na}^{+} /$glucose cotransporter in lactating bovine gastrointestinal tract. J. Anim. Sci. 76:29212929.

Zhao, F.-Q., E. R. Okine, and J. J. Kennelly. 1999. Glucose transporter gene expression in bovine mammary gland. J. Anim. Sci. $77: 2517-2522$. 